Bull. Austral. Math. Soc.

VOL. $69(2004)$ [161-171]

\title{
ON TENSOR FACTORISATION FOR REPRESENTATIONS OF FINITE GROUPS
}

\author{
Emanuele Pacifici
}

\begin{abstract}
We prove that, given a quasi-primitive complex representation $D$ for a finite group $G$, the possible ways of decomposing $D$ as an inner tensor product of two projective representations of $G$ are parametrised in terms of the group structure of $G$. More explicitly, we construct a bijection between the set of such decompositions and a particular interval in the lattice of normal subgroups of $G$.
\end{abstract}

\section{INTRODUCTION}

Throughout the whole paper, all the groups generically denoted by $G$ (or $H$ ) are meant to be finite, and all the representations will be finite dimensional representations over the complex field, although $\mathbb{C}$ can be safely replaced with any algebraically closed field of characteristic zero.

It is well known that, given an irreducible representation $D$ for a group $G$, a good understanding of $D$ is achieved if it is possible to find (and describe) a subgroup $H$ and a representation $T$ for it, such that $D$ is induced by $T$ from $H$. An effective method for recognising such a pair is provided by Clifford's Theorem $([1,11.1])$, iterated applications of which yield a pair $(H, T)$ such that $D$ is induced by $T$ from $H$, and $T$ is a quasiprimitive representation of $H$ (recall that an irreducible representation of a finite group is called quasi-primitive if its restriction to any normal subgroup has pairwise equivalent irreducible constituents). In view of that, understanding the structure of quasi-primitive representations appears as a crucial issue in Representation Theory.

Now, let $D$ be a faithful quasi-primitive representation for $G$. Although such a representation can still be induced from a proper subgroup of $G$ (but a result due to Berger excludes this possibility if $G$ is solvable (see $[7,11.33]$ ), there seems to be no general method to exploit further the additive structure of $D$, and it appears natural to investigate $D$ from the point of view of its 'multiplicative' structure. In particular, our aim in this paper is to control and parametrise, in terms of the group structure of $G$, all the possible ways of decomposing $D$ as an inner tensor product of two projective

Received 22nd September, 2003

I wish to express my gratitude to Laci Kovács, who pointed out to me the problem considered in this paper. His advice, during many fruitful and pleasant discussions, has been fundamental.

Copyright Clearance Centre, Inc. Serial-fee code: 0004-9727/04 \$A2.00+0.00. 
representations of $G$. With the further hypothesis that the restriction of $D$ to the Fitting subgroup $F$ of $G$ is irreducible, the main result of this paper (Corollary 2.8, which follows from the more general Theorem 2.6) shows essentially that there is an explicit bijection between the set of two-factors decompositions of $D$ and the set of normal subgroups of $G$ lying between the centre $Z$ and $F$. The assumption of faithfulness for $D$ was added only for ease of statements in this Introduction, but the hypothesis of irreducibility on the restriction $D \downarrow_{F}$ is serious: it excludes from consideration all (nonabelian) groups in which the Fitting subgroup is central (in particular, it excludes all nonabelian simple groups, for which the problem has an entirely different character that is beyond the present discussion). Of course, when $F$ is central, one cannot expect it to contain much information about factorisations of representations which map it to scalars. If $F$ is noncentral and $D \downarrow_{F}$ is reducible, a well known result (stated here as Lemma 2.1) gives a tensor factorisation for $D$ such that one factor represents $F$ irreducibly and the other just by scalars.

Since Corollary 2.8 describes how the projective-equivalence class of $D$ factorises, it can be conveniently translated into the language of characters, and this is done in Theorem 3.2. Finally, the example in Section 4 shows that the hypothesis of irreducibility for $D \downarrow_{F}$ can not be dropped (nor weakened along one particular line) even if $D$ is assumed primitive instead of quasi-primitive.

Tensor factorisation of quasi-primitive representations has been extensively studied, from the point of view of characters, in [3] (which in turn generalises some results from [4] and [8]); in that paper the authors prove that, given a quasi-primitive character $\chi$ of a group $G$, there exists an essentially unique 'admissible set of prime characters' which provides a factorisation for $\chi$. It is worth stressing that the aim of such a result is different from ours, as the problem of describing all factorisations is not considered; indeed, in some cases (even when the group is solvable) a 'prime character' could be factorised, but no factorisation is yelded by those methods. At any rate, taking in account that a prime character fits the hypotheses of Theorem 3.2 (at least in a solvable context), the present approach reveals some interactions with previous works on this subject.

\section{Projective Representations}

In this section we recall some basic definitions and results concerning projective (complex) representations, and we establish some notations and conventions.

Let $G$ be a group, $n$ a positive integer, and $P$ a map from $G$ to $G L(n, \mathbb{C})$. If there exists a map $\alpha$, from $G \times G$ to $\mathbb{C}^{\times}$, such that $P\left(g_{1}\right) P\left(g_{2}\right)=\alpha\left(g_{1}, g_{2}\right) P\left(g_{1} g_{2}\right)$ holds for all $g_{1}, g_{2}$ in $G$, then $P$ is called a projective representation of degree $n$ for $G$. The map $\alpha$ is the factor set of $P$. and it is uniquely determined by $P$. It is clear that any representation is a projective representation; sometimes, for the sake of emphasis, a representation in the classical sense will be called (following [9]) a genuine representation. 
If $P_{1}$ and $P_{2}$ are projective representations of degree $n$ for $G$, then we say that they are equivalent if there exist a matrix $A$ in $G L(n, \mathbb{C})$, and a map $\beta: G \rightarrow \mathbb{C}^{\times}$, such that $P_{2}(g)=\beta(g) A^{-1} P_{1}(g) A$ for all $g$ in $G$. This defines an equivalence relation on the set of projective representations of $G$, and we shall denote by $[P]$ the class of a projective representation $P$ modulo this equivalence relation.

Let $P$ be a projective representation of degree $n$ for $G$, and $V$ an $n$-dimensional vector space over $\mathbb{C}$. We say that $P$ is irreducible if the only subspaces of $V$ invariant under the action of the set of matrices $P(G)$ are the zero space and $V$ itself.

REMARK 1.1. If $\bar{P}$ is the composite of a projective representation $P$ of degree $n$ for $G$ with the natural homomorphism $\pi$ which maps $G L(n, \mathbb{C})$ onto $P G L(n, \mathbb{C})$, then $\bar{P}$ is a homomorphism from $G$ to $P G L(n, \mathbb{C})$. Conversely, if $\tau: G \rightarrow P G L(n, \mathbb{C})$ is a homomorphism, a projective representation of degree $n$ for $G$ arises in a natural way (as soon as we choose a transversal for $Z(G L(n, \mathbb{C}))$ in the full preimage under $\pi$ of $\tau(G)$; such a choice is however not relevant up to equivalence).

It is also useful to introduce a concept of equivalence for homomorphisms to projective general linear groups: let $\tau_{1}$ and $\tau_{2}$ be homomorphisms of $G$ to $\operatorname{PGL}(n, \mathbb{C})$; we say that $\tau_{1}$ and $\tau_{2}$ are equivalent if there exists $A$ in $G L(n, \mathbb{C})$ such that $\tau_{2}(g)=\tau_{1}(g)^{\pi(A)}$ for all $g$ in $G$ (and, when we write the symbol ' $\simeq$ ' between two such homomorphisms, we refer to this kind of equivalence). If we choose now two projective representations $P_{1}$ and $P_{2}$ of $G$ with $\overline{P_{1}}=\tau_{1}$ and $\overline{P_{2}}=\dot{\tau}_{2}$, it is clear that $\tau_{1}$ and $\tau_{2}$ are equivalent if and only if $P_{1}$ and $P_{2}$ are so.

Projective representations play a fundamental role in the present context because it is possible to construct inner tensor products with them, and such a product may yield a genuine representation: if $P_{1}, P_{2}$ are projective representations for the group $G$, with factor sets $\alpha_{1}, \alpha_{2}$ and degrees $n, m$ respectively, then the map $P_{1} \otimes P_{2}: G \rightarrow G L(n m, \mathbb{C})$ defined by $\left(P_{1} \otimes P_{2}\right)(g):=P_{1}(g) \otimes P_{2}(g)$ for all $g$ in $G$, is a projective representation of $G$ whose factor set is the pointwise product of $\alpha_{1}$ and $\alpha_{2}$ (the symbol ' $\otimes$ ' between two matrices denotes the usual Kronecker product); this projective representation is called the inner tensor product of $P_{1}$ and $P_{2}$.

We can now introduce some more notations.

DEFinition 1.2: Let $G$ be a group, and $P_{1}, P_{2}$ projective representations of $G$; we denote by $\overline{P_{1}} \otimes \overline{P_{2}}$ the homomorphism $\overline{P_{1} \otimes \bar{P}_{2}}$.

To avoid any confusion arising from the fact that two different concepts of equivalence are floating around for genuine representations (depending on whether they are regarded as genuine or as projective representations), we shall emphasise the distinction, when needed, saying that two representations are genuine-equivalent if they are equivalent in the classical sense, whereas we shall call them projective-equivalent if they are equivalent only (in principle) as projective representations. It is clear that two genuine 
representations $D_{1}$ and $D_{2}$ of $G$ are projective-equivalent if and only if there exists a 1-dimensional representation $\lambda$ of $G$ such that $D_{1}$ and $\lambda \otimes D_{2}$ are genuine-equivalent.

\section{FActorisation of REpresentations}

The main result of the paper, which is 2.8 , follows as a corollary of Theorem 2.6. Before proving it we need to prepare the setting with some lemmas; the first of them is a well known result, and here it is only stated (see [6, 21.1(a) and 21.2] for a proof). As the last remark about notations, in what follows we shall denote by $I_{e}(e$ being a positive integer) the trivial complex representation of degree $e$, as well as the $e$-dimensional identity matrix over $\mathbb{C}$.

LemMA 2.1. Let $G$ be a group, $N$ a normal subgroup of $G, T$ an irreducible representation of $N$, and $D$ an irreducible representation of $G$ such that $D \downarrow_{N}=I_{e} \otimes T$. Then there exist projective representations $P_{1}$ and $P_{2}$ of $G$ such that

(a) $D(g)=P_{1}(g) \otimes P_{2}(g)$ for all $g$ in $G$,

(c) $P_{1}(x)=I_{e}$ and $P_{2}(x)=T(x)$ for all $x$ in $N$,

(c) $T(x)^{P_{2}(g)}=T\left(x^{g}\right)$ for all $x$ in $N$ and $g$ in $G$.

Observe that, if $T^{\prime}$ is a representation of $N$ which is genuine-equivalent to $T$, and $P, P^{\prime}$ are projective representations of $G$ which satisfy condition (c) of 2.1 for $T$ and $T^{\prime}$ respectively, then (by Schur's Lemma) $P$ and $P^{\prime}$ are projective-equivalent.

Assume now that the group $G$ is a central product of the subgroups $A$ and $B$, that is, $G=A B$ with $[A, B]=1$. In this case $G$ can be identified with a quotient of the (external) direct product $A \times B$ and, by inflation, each representation of $G$ may be viewed as a representation of $A \times B$. In particular, by [5, 3.7.1], each irreducible representation of $G$ may be viewed as an outer tensor product $R \# S$ of some irreducible representation $R$ of $A$ and some irreducible representation $S$ of $B$, such that $R \downarrow_{A \cap B}=\xi \otimes I_{\operatorname{deg} R}$ and $S \downarrow_{A \cap B}=\xi \otimes I_{\operatorname{deg} S}$ for a suitable 1-dimensional representation $\xi$ of $A \cap B$ (recall that $R \# S$ maps, by definition, an element $(a, b)$ of $A \times B$ to the matrix $R(a) \otimes S(b))$. Conversely, if $R$ and $S$ are irreducible representations of $A$ and $B$ satisfying this condition for some $\xi$, then $R \# S$ may be viewed as an (irreducible) representation of $G$.

LEMMA 2.2. Let the group $G$ be a central product of the subgroups $A$ and $B$. Then the following properties hold:

(a) if $R$ is an irreducible genuine representation for one of the central factors, say $A$, then there exists a unique homomorphism $\varrho$, from $G$ to $P G L(\operatorname{deg} R, \mathbb{C})$, such that $\varrho \downarrow_{A}=\bar{R}$ and $B \leqslant \operatorname{ker} \varrho$,

(b) let $R$ and $S$ be irreducible genuine representations, for $A$ and $B$ respectively, such that $R \# S$ is a representation for $G$. If $\varrho$ and $\sigma$ are homomorphisms as in (a) for $R$ and $S$, then we have $\overline{R \# S}=\varrho \otimes \sigma$. 
Proof OF (a): For each element $g$ of $G$, consider an element $a$ in $A$ and an element $b$ in $B$ such that $g=a b$, and define $\varrho(g)$ to be $\bar{R}(a)$. It is routine to check that $\varrho$ is well defined and satisfies the required conditions.

Proof of (b): Let $X$ and $Y$ be projective representations of $G$ such that $\bar{X}=\varrho$ and $\bar{Y}=\sigma$ (see Remark 1.1); following Definition 1.2, $\varrho \otimes \sigma$ is the homomorphism $\overline{X \otimes Y}$. Now, given an element $g$ in $G$, let $a$ and $b$ be elements of $A$ and $B$ respectively, such that $g=a b$; since we get $\bar{X}(g)=\varrho(g)=\bar{R}(a)$, and $\bar{Y}(g)=\sigma(g)=\bar{S}(b)$, claim (b) follows simply by applying the definitions.

It will be convenient to have a temporary name for a class of groups which will play an important role in our proofs (but will not appear in our conclusions).

DEFINITION 2.3: Let $F$ be a finite group; we say that $F$ is a good group if $Z(F)$ is cyclic and $F / Z(F)$ is Abelian of squarefree exponent.

The reason these groups are so important here is the following lemma; for a proof, see $[3,1.4]$.

LEMMA 2.4. Let $G$ be a group with Fitting subgroup $F$ and centre $Z$. If $G$ has a faithful quasi-primitive representation, then $F / Z$ is an Abelian group of squarefree exponent.

The relevant properties of good groups will be outlined next.

LEMMA 2.5. Let $F$ be a good group, and let $Z$ denote its centre; then the following properties hold:

(a) if $K$ is a subgroup of $F$ such that $Z(K)=Z$, then $F$ is the (central) product of $K$ and $C_{F}(K)$;

(b) if $P$ is an irreducible projective representation of $F$ with $Z \leqslant \operatorname{ker} \bar{P}$, then we have $(\operatorname{deg} P)^{2}=|F: \operatorname{ker} \bar{P}|$;

(c) if $D$ is a faithful irreducible representation of $F$, and $\bar{D} \simeq \overline{P_{1}} \otimes \overline{P_{2}}$ where $P_{1}$ and $P_{2}$ are projective representations of $F$ (here equivalence is in the sense of Remark 1.1), then we have $F=\operatorname{ker} \overline{P_{1}} \cdot \operatorname{ker} \overline{P_{2}}$;

(d) with the same assumptions as in (c), if $K$ is the kernel of $\overline{P_{1}}$, then $Z(K)$ coincides with $Z$; moreover, denoting by $L$ the kernel of $\bar{P}_{2}$, we have $L$ $=C_{F}(K)$;

(e) with the same assumptions as in (c), there exist genuine representations $R$ and $S$, of $K:=\operatorname{ker} \overline{P_{1}}$ and $L:=\operatorname{ker} \overline{P_{2}}=C_{F}(K)$ respectively, such that $R \# S$ is a representation of $F$ which is genuine-equivalent to $D$. Moreover, we have $\overline{P_{1}}=\sigma$ and $\overline{P_{2}}=\varrho$, where $\varrho$ and $\sigma$ are the homomorphisms linked to $R$ and $S$ which were constructed in $2.2(\mathrm{a})$, so that we have $\bar{P}_{1} \downarrow_{L}=\bar{S}$ and $\bar{P}_{2} \downarrow_{K}=\bar{R}$. This implies that $P_{1} \downarrow_{L}$ is a genuine representation of $L$ up to multiplying it by a suitable map from $L$ to $\mathbb{C}^{\times}$, and $P_{2}$ behaves similarly with respect to $K$. 
Proof of (a): Let $Q$ be a (nontrivial) Sylow subgroup of $F$, say for the prime $q$. We claim first that $Z(Q)=Z(K \cap Q)$. To see this, note that $Z(Q) \leqslant Z$ because $F$ is nilpotent; as $Z \leqslant K$, this proves that $Z(Q)$ is contained in $K \cap Q$, and then of course $Z(Q) \leqslant Z(K \cap Q)$. Conversely, $Z(K \cap Q)$ lies in $Z(K)=Z$ because $K$ is nilpotent and $K \cap Q$ is the Sylow $q$-subgroup of $K$, and hence $Z(K \cap Q) \leqslant Z(Q)$.

It follows that $(K \cap Q) \cap C_{Q}(K \cap Q)=Z(Q)$, hence the product of $(K \cap Q) / Z(Q)$ and $C_{Q}(K \cap Q) / Z(Q)$ is a direct product. We want to show next that this is all of $Q / Z(Q)$. Suppose that $|(K \cap Q) / Z(Q)|=q^{n}$ so $(K \cap Q) / Z(Q)$ is an $n$-dimensional vector space over $G F(q)$, and choose a basis $\left\{Z(Q) x_{1}, \ldots, Z(Q) x_{n}\right\}$ for it. We have $C_{Q}(K \cap Q)=\bigcap_{i=1}^{n} C_{Q}\left(x_{i}\right)$. Using that $F / Z$ is Abelian of squarefree exponent, it is easy to see that each map $\alpha_{i}: Q \rightarrow Z$ defined by $\alpha_{i}(x)=\left[x_{i}, x\right]$ is a homomorphism whose kernel is $C_{Q}\left(x_{i}\right)$ and whose image has exponent dividing $q$. Since $Z$ is cyclic, we conclude that $\left|Q: C_{Q}\left(x_{i}\right)\right| \leqslant q$, thus $\left|Q / C_{Q}(K \cap Q)\right| \leqslant q^{n}$. Thus the dimension of $(K \cap Q) / Z(Q)$ $\times C_{Q}(K \cap Q) / Z(Q)$ (as a vector space over $G F(q)$ ) is at least the dimension of $Q / Z(Q)$, and our claim follows.

Finally, let $\left\{Q_{1}, \ldots, Q_{h}\right\}$ be the set of (nontrivial) Sylow subgroups of $F$ : we have

$$
F=\left(K \cap Q_{1}\right) C_{Q_{1}}\left(K \cap Q_{1}\right) \cdots\left(K \cap Q_{h}\right) C_{Q_{h}}\left(K \cap Q_{h}\right)=K C_{F}(K),
$$

as desired.

Proof of (b): Since $P$ is an irreducible projective representation such that $\operatorname{ker} \bar{P}$ contains $Z$, we have that $\bar{P}(F)$ is an irreducible Abelian subgroup of $P G L(\operatorname{deg} P, \mathbb{C})$ (which is of course isomorphic to $F / \operatorname{ker} \bar{P})$; if $M$ is the preimage of $\bar{P}(F)$ in $G L(\operatorname{deg} P, \mathbb{C}$ ) under the natural homomorphism, we have $Z(G L(\operatorname{deg} P, \mathbb{C})) \leqslant Z(M)$ but, since $M$ is irreducible, equality holds. Moreover, $M$ is nilpotent of class 2, so that $(\operatorname{deg} P)^{2}$ $=|M / Z(M)|=|\bar{P}(F)|$ (this is not hard to prove; see for example $[2,4.3]$ ) and our claim follows.

Proof of (c): Since $D$ is faithful, we have $\operatorname{ker} \bar{P}_{1} \cap \operatorname{ker} \bar{P}_{2}=Z$ (this is easily seen, as the Kronecker product of two matrices is a scalar matrix if and only if the factors are scalar matrices), so that $\operatorname{ker} \bar{P}_{1} / Z \cdot \operatorname{ker} \bar{P}_{2} / Z=\operatorname{ker} \bar{P}_{1} / Z \times \operatorname{ker} \bar{P}_{2} / Z ;$ this is a subgroup of the Abelian group with squarefree exponent $F / Z$, hence it suffices to show that $l\left(\operatorname{ker} \bar{P}_{1} / Z\right)+l\left(\operatorname{ker} \bar{P}_{2} / Z\right)=l(F / Z)$, where $l(G)$ denotes the composition length of a given group $G$.

Since we have $l\left(\operatorname{ker} \bar{P}_{i} / Z\right)=l(F / Z)-l\left(F / \operatorname{ker} \bar{P}_{i}\right)$, what we want to show is $l(F / Z)$ $=l\left(F / \operatorname{ker} \bar{P}_{1}\right)+l\left(F / \operatorname{ker} \bar{P}_{2}\right)$. Let $H$ be the set $\left\{\left(\bar{P}_{1}(x), \overline{P_{2}}(x)\right): x \in F\right\}$, which is indeed a subgroup of the (external) direct product $\overline{P_{1}}(F) \times \overline{P_{2}}(F)$, and let $\varphi: H \rightarrow P G L(\operatorname{deg} D, \mathbb{C})$ be the map defined by $\varphi\left(\left(\overline{P_{1}}(x), \overline{P_{2}}(x)\right)\right):=\overline{P_{1}(x) \otimes P_{2}(x)}$. It is easily seen that $\varphi$ is a monomorphism, and therefore $H \simeq \varphi(H) \simeq \bar{D}(F)$ holds; but 
now, by part (b), we have

$$
|\bar{D}(F)|=(\operatorname{deg} D)^{2}=\left(\operatorname{deg} P_{1}\right)^{2}\left(\operatorname{deg} P_{2}\right)^{2}=\left|\bar{P}_{1}(F)\right|\left|\bar{P}_{2}(F)\right|,
$$

thus $\bar{D}(F) \simeq \bar{P}_{1}(F) \times \bar{P}_{2}(F)$, and the claim is proved (as $F / Z \simeq \bar{D}(F)$ and $F / \operatorname{ker} \bar{P}_{i}$ $\left.\simeq \overline{P_{i}}(F)\right)$.

Proof of (d): By part (c) we have $F=K L$. Let us now prove that $[K, L]=1$. Denoting by $x$ an element of $K$ and by $y$ an element of $L$, there exist $A$ in $G L(\operatorname{deg} D, \mathbb{C})$ and $\lambda, \mu$ in $\mathbb{C}^{\times}$such that

$$
A^{-1} D(x) A=\lambda I_{\operatorname{deg} P_{1}} \otimes P_{2}(x) \text { and } A^{-1} D(y) A=P_{1}(y) \otimes \mu I_{\operatorname{deg} P_{2}} ;
$$

it is now clear that $[D(K), D(L)]=1$, and the faithfulness of $D$ yields what we wanted. Of course now we have $Z(K)=Z$ and, since the conditions $F=K L, L \leqslant C_{F}(K)$ and $K \cap L=K \cap C_{F}(K)=Z$ hold, we conclude that $L=C_{F}(K)$.

Proof of (e): By assumption, there exist an element $A$ in $G L(\operatorname{deg} D, \mathbb{C})$ and a map $\lambda$ from $F$ to $\mathbb{C}^{\times}$such that $\lambda(f) A^{-1} D(f) A=P_{1}(f) \otimes P_{2}(f)$ holds for all $f$ in $F$. Now, for all $k$ in $K$, we get $\lambda(k) A^{-1} D(k) A=\mu(k) I_{\operatorname{deg} P_{1}} \otimes P_{2}(k)$, where $\mu$ is a map from $K$ to $\mathbb{C}^{\times}$; defining $R(k)$ as $\lambda(k)^{-1} \mu(k) P_{2}(k)$ we get $A^{-1} D(k) A=I_{\operatorname{deg} P_{1}} \otimes R(k)$, so that $R$ is a genuine representation of $K$. Similarly, a genuine representation $S$ for $L$ can be defined so that, for all $l$ in $L$, we have $A^{-1} D(l) A=S(l) \otimes I_{\operatorname{deg} P_{2}}$. Now, for every element $f$ in $F$, we can choose $k$ in $K$ and $l$ in $L$ such that $f=k l$, obtaining

$$
\begin{aligned}
A^{-1} D(f) A & =\left(A^{-1} D(k) A\right)\left(A^{-1} D(l) A\right) \\
& =\left(I_{\operatorname{deg} P_{1}} \otimes R(k)\right)\left(S(l) \otimes I_{\operatorname{deg} P_{2}}\right) \\
& =S(l) \otimes R(k) \\
& =(S \# R)(f),
\end{aligned}
$$

so that $D$ is genuine-equivalent to $R \# S$ (swapping the factors does not change the equivalence type), and both of $R$ and $S$ are irreducible. Finally, recalling that $\sigma$ is defined by $\sigma(f):=\bar{S}(l)$, and observing that we have $\bar{S}(l)=\bar{P}_{1}(l)=\bar{P}_{1}(f)$, we conclude that $\sigma=\overline{P_{1}}$; in an entirely similar way we also get $\varrho=\overline{P_{2}}$.

We are now in a position to prove the main results of the paper.

THEOREM 2.6. Let $H$ be a group, and let $F$ be a good group such that $F$ is a normal subgroup of $H$ and $C_{H}(F)$ is contained in $F$. Let $D$ be a faithful representation of $H$ such that $D \downarrow_{F}$ is irreducible. Then there exists a bijection between the set of all the pairs $\left(\left[P_{1}\right],\left[P_{2}\right]\right)$, where $P_{1}, P_{2}$ are projective representations of $H$ such that $\bar{D} \simeq \overline{P_{1}} \otimes \overline{P_{2}}$, and the set of normal subgroups $K$ of $H$ such that $K \leqslant F$ and $Z(K)=Z(H)$ hold. In particular, such a bijection can be constructed by mapping $\left(\left[P_{1}\right],\left[P_{2}\right]\right)$ to $K:=\operatorname{ker}\left(\overline{P_{1}} \downarrow_{F}\right)$ and, $K$ being so defined, we also have $\operatorname{ker}\left(\bar{P}_{2} \downarrow_{F}\right)=C_{F}(K)$. 
Proof: We shall denote by $Z$ the centre of $H$; also, we shall denote by $\mathcal{F}_{D}$ the set of all the pairs $\left(\left[P_{1}\right],\left[P_{2}\right]\right)$, where $P_{1}, P_{2}$ are projective representations of $H$ such that $\bar{D} \simeq \overline{P_{1}} \otimes \overline{P_{2}}$, and by $\mathcal{S}$ the set of normal subgroups $K$ of $H$ such that $K$ lies in $F$ and $Z(K)=Z$. Next we observe that, since $D$ is faithful and its restriction to $F$ is irreducible, we have $Z(F) \leqslant Z$; but $F$ contains its own centraliser in $H$, therefore $Z(F)$ coincides with $Z$.

Now, as the first step in the proof, we shall construct a map $\alpha$ from $\mathcal{F}_{D}$ to $\mathcal{S}$ : consider an element $\left(\left[P_{1}\right],\left[P_{2}\right]\right)$ in $\mathcal{F}_{D}$ and define $\alpha\left(\left(\left[P_{1}\right],\left[P_{2}\right]\right)\right)$ as the kernel of $\bar{P}_{1} \downarrow_{F}$. Since equivalent projective representations yield homomorphisms (to the relevant projective general linear group) which have the same kernel, the 'value' $\alpha\left(\left(\left[P_{1}\right],\left[P_{2}\right]\right)\right)$ does not depend on the choice of representatives for the classes $\left[P_{1}\right]$ and $\left[P_{2}\right]$; moreover, denoting by $K$ the kernel of $\bar{P}_{1} \downarrow_{F}$, Lemma 2.5 (d) tells us that $Z(K)=Z$, and certainly we have $K \leqslant F$ and $K \unlhd H$. The discussion above shows that $\alpha$ is actually a map from $\mathcal{F}_{D}$ to $\mathcal{S}$. Also, again by Lemma $2.5(\mathrm{~d})$, we get $C_{F}(K)=\operatorname{ker}\left(\bar{P}_{2} \downarrow_{F}\right)$.

As the second step we shall show that, given an element $K$ of $\mathcal{S}$, there exists a unique element $\left(\left[P_{1}\right],\left[P_{2}\right]\right)$ in $\mathcal{F}_{D}$ such that $\operatorname{ker}\left(\bar{P}_{1} \downarrow_{F}\right)=K$; this will prove that $\alpha$ is a bijection. So, let us start from an element $K$ in $\mathcal{S}$; by Lemma $2.5(\mathrm{a})$ we get $F=K C_{F}(K)$. If we denote by $\chi$ the character afforded by $D$, we have $\chi \downarrow_{F}=\varphi$ for some $\varphi$ in $\operatorname{Irr} F$; now $K$ is a normal subgroup of $F$ and, if $\vartheta$ is an irreducible constituent of $\varphi \downarrow_{K}$, then the inertia subgroup $I_{F}(\vartheta)$ is all of $F$. We conclude that $\chi \downarrow_{K}=e \vartheta$, where $e$ is a positive integer, hence we can assume $D \downarrow_{K}=I_{e} \otimes T$ where $T$ is an irreducible representation of $K$ affording the character $\vartheta$. We are now in a position to apply Lemma 2.1 , which ensures the existence of an element $\left(\left[P_{1}\right],\left[P_{2}\right]\right)$ in $\mathcal{F}_{D}$ with the properties that $K$ is contained in $\operatorname{ker}\left(\bar{P}_{1} \downarrow_{F}\right)$, $\operatorname{deg} P_{2}=\operatorname{deg} T$ and $T\left(x^{h}\right)=T(x)^{P_{2}(h)}$ for all $x$ in $K$ and $h$ in $H$. We want now to prove that $K$ coincides with $\operatorname{ker}\left(\bar{P}_{1} \downarrow_{F}\right)$. Let $x$ be in $\operatorname{ker}\left(\bar{P}_{1} \downarrow_{F}\right)$, and let $k, c$ be elements, of $K$ and $C_{F}(K)$ respectively, such that $x=k c$ (again we are using Lemma 2.5(a)). Since $K$ is contained in $\operatorname{ker}\left(\bar{P}_{1} \downarrow_{F}\right), c$ lies in $\operatorname{ker}\left(\bar{P}_{1} \downarrow_{F}\right)$ as well, so that we have $D(c)=\mu I_{e} \otimes P_{2}(c)$ for some $\mu$ in $\mathbb{C}^{\times}$; moreover, $c$ is in $C_{F}(K)$, hence $T(y)^{P_{2}(c)}=T\left(y^{c}\right)=T(y)$ holds for all $y$ in $K$, so that $P_{2}(c)$ is a scalar matrix, say $\nu I_{\operatorname{deg} T}$ for some $\nu$ in $\mathbb{C}^{\times}$. We conclude that $D(c)$ is given by the Kronecker product of two scalar matrices, therefore $c$ lies in $Z$ (by the faithfulness of $D$ ) and the claim follows.

To complete the second step of the proof, we need to show that $K$ determines uniquely an element $\left(\left[P_{1}\right],\left[P_{2}\right]\right)$ of $\mathcal{F}_{D}$ with the property that $\operatorname{ker}\left(\bar{P}_{1} \downarrow_{F}\right)=K$. For this purpose observe that, since $\bar{D} \simeq \overline{P_{1}} \otimes \overline{P_{2}}$, there exist $A$ in $G L(\operatorname{deg} D, \mathbb{C})$ and a map $\lambda$ from $H$ to $\mathbb{C}^{\times}$such that $A^{-1} D(h) A=(\lambda(h))^{-1} P_{1}(h) \otimes P_{2}(h)$ holds for all $h$ in $H$. Moreover, we get $A^{-1} D(k) A=I_{\operatorname{deg} P_{1}} \otimes R(k)$ for all $k$ in $K$, where $R$ is the genuine irreducible representation of $K$ defined in the proof of Lemma 2.5(e). From $D\left(k^{h}\right)=D(k)^{D(h)}$ we obtain now $I_{\operatorname{deg} P_{1}} \otimes R\left(k^{h}\right)=I_{\operatorname{deg} P_{1}} \otimes R(k)^{P_{2}(h)}$, hence $R\left(k^{h}\right)=R(k)^{P_{2}(h)}$ for all $h$ in $H$ and $k$ in $K$. Since the genuine-equivalence type of $R$ is uniquely determined by $K$ 
and by the genuine-equivalence type of $D$, we conclude that the projective-equivalence type of $P_{2}$, that is, $\left[P_{2}\right]$, is uniquely determined by $K$ and by the genuine-equivalence type of $D$. Similarly, we see that $\left[P_{1}\right]$ is uniquely determined by $\operatorname{ker}\left(\bar{P}_{2} \downarrow_{F}\right)$, which is in turn determined by $K$, being its centraliser in $F$ (Lemma 2.5(d)), and by the genuineequivalence type of $D$.

Definition 2.7: Let $G$ be a group, and $D$ a representation of $G$. We define the subgroups $Z(D)$ and $F(D)$ of $G$ by $Z(D) / \operatorname{ker} D:=Z(G / \operatorname{ker} D)$ and $F(D) / \operatorname{ker} D:=$ $F(G / \operatorname{ker} D)$.

COROLlary 2.8. Let $G$ be a group, and $D$ a quasi-primitive representation of $G$ such that $D \downarrow_{F(D)}$ is irreducible. There is a bijection between the set of all the pairs $\left(\left[P_{1}\right],\left[P_{2}\right]\right)$, where $P_{1}$ and $P_{2}$ are projective representations of $G$ such that $\bar{D} \simeq \overline{P_{1}} \otimes \overline{P_{2}}$, and the interval $[Z(D), F(D)]$ in the lattice of normal subgroups of $G$. Such a bijection can be constructed by mapping $\left(\left[P_{1}\right],\left[P_{2}\right]\right)$ to $K:=\operatorname{ker}\left(\bar{P}_{1} \downarrow_{F(D)}\right)$ and, $K$ being so defined, we also have $\operatorname{ker}\left(\bar{P}_{2} \downarrow_{F(D)}\right)=\{x \in F(D):[x, K] \subseteq \operatorname{ker} D\}$.

Proof: Denoting by $X$ the kernel of $D$, consider the quotient group $\widehat{G}:=G / X$; if $\Delta$ is the representation of $\widehat{G}$ defined by $\Delta(X g):=D(g)$ for all $X g$ in $\widehat{G}$, we have that $\Delta$ is faithful and $\Delta \downarrow_{F(\widehat{G})}$ is irreducible, therefore $C_{\widehat{G}}(F(\widehat{G}))$ is in the centre of $\widehat{G}$ and, in particular, it lies in $F(\widehat{G})$. This implies $Z(\widehat{G})=Z(F(\widehat{G}))$ and, since $\Delta$ is quasi-primitive, we conclude that $F(\widehat{G})$ is a good group (see 2.4 ), obviously a normal subgroup of $\widehat{G}$. Now we are in a position to apply Theorem 2.6 , obtaining that there exists a bijection between the set $\mathcal{F}_{\Delta}$ of all the pairs $\left(\left[Q_{1}\right],\left[Q_{2}\right]\right)$, where $Q_{1}$ and $Q_{2}$ are projective representations of $\widehat{G}$ such that $\bar{\Delta} \simeq \overline{Q_{1}} \otimes \overline{Q_{2}}$, and the set of normal subgroups $\widehat{K}$ of $\widehat{G}$ such that $\widehat{K} \leqslant F(\widehat{G})$ and $Z(\widehat{K})=Z(\widehat{G})$. We also know that, if $\left(\left[Q_{1}\right],\left[Q_{2}\right]\right)$ corresponds to $\widehat{K}$ in the relevant bijection, then we have $\widehat{K}=\operatorname{ker}\left(\overline{Q_{1}} \downarrow_{F(\widehat{G})}\right)$ and $C_{F(\widehat{G})}(\widehat{K})=\operatorname{ker}\left(\overline{Q_{2}} \downarrow_{F(\widehat{G})}\right)$.

Consider now a projective representation $P$ of $G$ such that $X$ is contained in $\operatorname{ker} \bar{P}$; we can choose a projective representation $Q$ of $\widehat{G}$ such that $\bar{Q}(X g):=\bar{P}(g)$ for all $X g$ in $\widehat{G}$ and, associating $[P]$ with $[Q]$, we can easily construct a bijection between $\mathcal{F}_{\Delta}$ and the set of all the pairs $\left(\left[P_{1}\right],\left[P_{2}\right]\right)$, where $P_{1}$ and $P_{2}$ are projective representations of $G$ such that $\bar{D} \simeq \overline{P_{1}} \otimes \overline{P_{2}}$. Also, the natural correspondence between normal subgroups of $\widehat{G}$ and normal subgroups of $G$ containing $X$ provides, by restriction, a bijection between the set of normal subgroups $\widehat{K}$ of $\widehat{G}$ such that $\widehat{K} \leqslant F(\widehat{G})$ and $Z(\widehat{K})=Z(\widehat{G})$, and the interval $[Z(D), F(D)]$ in the lattice of normal subgroups of $G$; the proof can be now easily completed.

\section{FACTORISATION OF CHARACTERS}

We give next an interpretation of the discussion above in terms of characters.

Definition 3.1: Let $G$ be a group; we denote by $(\widetilde{G}, \pi)$ a Schur covering of $G$ (so that $\widetilde{G}$ is a Schur representation group for $G$; see [7], Chapter 11), and by $A$ the kernel 
of $\pi$, which is a central subgroup of $\widetilde{G}$; if $H$ is a subgroup of $G$, we define $\widetilde{H}$ as $\pi^{-1}(H)$.

If $\chi$ and $\psi$ are irreducible characters of $G$, we say that they are equivalent (and we write $\chi \simeq \psi$ ) if there exists $\lambda$ in $\operatorname{Irr} G$ such that $\lambda(1)=1$ and $\chi=\lambda \psi$. It is clear that, in this way, an equivalence relation on the $\operatorname{set} \operatorname{Irr} G$ is defined; we shall denote by $[\chi]$ the equivalence class of the character $\chi$ modulo this equivalence relation.

Finally, we define $Z(\chi)$ and $F(\chi)$ in analogy with Definition 2.7; observe that, if $\chi_{\text {inf }}$ is the character of $\widetilde{G}$ obtained from $\chi$ by inflation, we have $\widetilde{F(\chi)}=F\left(\chi_{\mathrm{inf}}\right)$.

THEOREM 3.2. Let $G$ be a group, and $\chi$ a quasi-primitive character of $G$ such that $\chi \downarrow_{F(x)}$ is irreducible. Then the following properties hold:

(a) if $N$ is a normal subgroup of $G$ with $Z(\chi) \leqslant N \leqslant F(\chi)$, then there exist characters $\varrho_{1}$ and $\varrho_{2}$ of $\widetilde{G}$ such that $\chi_{\mathrm{inf}} \simeq \varrho_{1} \varrho_{2}$ and $Z\left(\varrho_{1} \downarrow \widetilde{F(\chi)}\right)=\widetilde{N}$;

(b) let $\varrho_{1}, \varrho_{2}, \varrho_{3}$ and $\varrho_{4}$ be irreducible characters of $G$ such that $\chi \simeq \varrho_{1} \varrho_{2}$ and $\chi \simeq \varrho_{3} \varrho_{4}$; if $Z\left(\varrho_{1} \downarrow_{F(\chi)}\right)$ is the same as $Z\left(\varrho_{3} \downarrow_{F(\chi)}\right)$, then we have $\varrho_{1} \simeq \varrho_{3}$ and $\varrho_{2} \simeq \varrho_{4} ;$

(c) there is a bijection between the set of all the pairs $\left(\left[\varrho_{1}\right],\left[\varrho_{2}\right]\right)$, where $\varrho_{1}$ and $\varrho_{2}$ are characters of $\widetilde{G}$ such that $\chi_{\mathrm{inf}} \simeq \varrho_{1} \varrho_{2}$, and the interval $[Z(\chi), F(\chi)]$ in the lattice of normal subgroups of $G$. Such a bijection can be constructed by mapping $\left(\left[\varrho_{1}\right],\left[\varrho_{2}\right]\right)$ to the subgroup $N$ such that $Z\left(\varrho_{1} \downarrow \widetilde{F(x)}\right)=\tilde{N}$.

Proof of (a): Let $D$ be a representation of $G$ which affords $\chi ; D$ is quasi-primitive, its restriction to $F(D)=F(\chi)$ is irreducible, and $N$ is a normal subgroup of $G$ with $Z(D) \leqslant N \leqslant F(D)$; hence Corollary 2.8 yields that there exist projective representations $P_{1}$ and $P_{2}$ of $G$ such that $\bar{D} \simeq \bar{P}_{1} \otimes \bar{P}_{2}$ and $\operatorname{ker}\left(\bar{P}_{1} \downarrow_{F(D)}\right)=N$. As $(\widetilde{G}, \pi)$ is a Schur covering for $G$, we can find genuine representations $D_{1}$ and $D_{2}$ of $\widetilde{G}$, together with maps $\xi_{1}$ and $\xi_{2}$ from $\tilde{G}$ to $\mathbb{C}^{\times}$, such that $\xi_{1}(x) D_{1}(x)=P_{1}(A x)$ and $\xi_{2}(x) D_{2}(x)=P_{2}(A x)$ for all $x$ in $\widetilde{G}$ (here we are identifying $G$ with $\tilde{G} / A$ ); it is now easy to see that $D$, viewed by inflation as a representation of $\widetilde{G}$, is projective-equivalent to $D_{1} \otimes D_{2}$. We conclude that, denoting by $\varrho_{1}$ and $\varrho_{2}$ the characters of $\widetilde{G}$ afforded by $D_{1}$ and $D_{2}$, we get $\chi_{\text {inf }} \simeq \varrho_{1} \varrho_{2}$; moreover, it is easily checked that $Z\left(\varrho_{1} \downarrow \widetilde{F(x)}\right)$ coincides with $\tilde{N}$.

.Proof of (b): Let $D_{i}$ be a representation which affords $\varrho_{i}$, for $i$ in $\{1,2,3,4\}$; we have $\bar{D} \simeq \bar{D}_{1} \otimes \bar{D}_{2}$ and $\bar{D} \simeq \bar{D}_{3} \otimes \bar{D}_{4}$; moreover,

$$
\operatorname{ker}\left(\bar{D}_{1} \downarrow_{F(D)}\right)=Z\left(\varrho_{1} \downarrow_{F(x)}\right)=Z\left(\varrho_{3} \downarrow_{F(x)}\right)=\operatorname{ker}\left(\bar{D}_{3} \downarrow_{F(D)}\right)
$$

holds. By Corollary 2.8 we conclude that $\bar{D}_{1} \simeq \bar{D}_{3}$ and $\bar{D}_{2} \simeq \bar{D}_{4}$, so that the claim follows.

Proof of (c): This follows at once by the two previous statements.

\section{A final Remark}

Let $G$ be a group, and $P, Q, R$ projective representations of $G$ such that $\bar{P} \otimes \bar{Q}$ $\simeq \bar{P} \otimes \bar{R}=: \bar{D} ;$ if $D$ happens to be a genuine quasi-primitive representation of $G$ whose 
restriction to $F(D)$ is irreducible, then it follows from Corollary 2.8 that $Q$ and $R$ are equivalent (and therefore we have, under the right assuptions, a 'cancellation law').

Even this claim fails if we weaken the hypothesis of irreducibility for $D \downarrow_{F(D)}$, assuming only, for instance, that the restriction of $D$ to $F^{*}(D)$ is irreducible (here $F^{*}(D)$ denotes the preimage, under the natural homomorphism, of the generalised Fitting subgroup of $G /$ ker $D$ ). Consider for example $G=A_{9}$; if we denote by $P$ the 8-dimensional irreducible representation of $G$, by $Q$ and $R$ the two 21-dimensional irreducible representations of $G$ (which are inequivalent), and by $D$ the 168-dimensional irreducible representation of $G$, we see that $D$ is quasi-primitive (indeed primitive) and of course irreducible when restricted to $F^{*}(G)$. Moreover, $D$ is genuine-equivalent to both of $P \otimes Q$ and $P \otimes R$, and therefore we have $\bar{D} \simeq \bar{P} \otimes \bar{Q} \simeq \bar{P} \otimes \bar{R}$. But it is clear that $Q$ and $R$ are not equivalent, even in a projective sense.

\section{REFERENCES}

[1] C.W. Curtis and I. Reiner, Methods of representation theory I (J. Wiley and Son, New York, 1981).

[2] J.D. Dixon, The structure of linear groups (Van Nostrand Reinhold, London, 1971).

[3] P.A. Ferguson and A. Turull, 'Prime characters and factorizations of quasi-primitive characters', Math. Z. 190 (1985), 583-604.

[4] D. Gajendragadkar, 'A characteristic class of characters of finite $\pi$-separable groups', $J$. Algebra 59 (1979), 237-259.

[5] D. Gorenstein, Finite groups (Harper \& Row, New York, 1968).

[6] B. Huppert, Character theory of finite groups (De Gruyter, Berlin, 1998).

[7] I.M. Isaacs, Character theory of finite groups (Academic Press, New York, 1976).

[8] I.M. Isaacs, 'Primitive characters, normal subgroups and M-Groups', Math. Z. 177 (1981), 267-284.

[9] L.G. Kovács, 'On tensor induction of group representations', J. Aust. Math. Soc. Ser. A 49 (1990), 486-501.

Dipartimento di Matematica 'Ulisse Dini'

Università degli Studi di Firenze

viale Morgagni 67-A

50134 Firenze

Italy

e-mail: pacifici@math.unifi.it 